\title{
Variations
}

Variations

Revue internationale de théorie critique

$20 \mid 2017$

Expériences oppositionnelles

\section{La bureaucratie liquide}

A propos de la lutte contre la loi travail et le principe Macron

\section{Alexander Neumann}

\section{(2) OpenEdition}

Journals

Édition électronique

URL : http://journals.openedition.org/variations/810

DOI : 10.4000/variations.810

ISSN : 1968-3960

Éditeur

Les amis de Variations

Référence électronique

Alexander Neumann, « La bureaucratie liquide », Variations [En ligne], 20 | 2017, mis en ligne le 25 avril 2017, consulté le 25 février 2021. URL : http://journals.openedition.org/variations/810 ; DOI : https:// doi.org/10.4000/variations.810

Ce document a été généré automatiquement le 25 février 2021.

Les ami•e•s de Variations 


\title{
La bureaucratie liquide
}

\author{
A propos de la lutte contre la loi travail et le principe Macron
}

\section{Alexander Neumann}

$1 \mathrm{Vu}$ depuis ce centre décentré qu'est Paris, la résistance contre la mondialisation capitaliste se manifeste dans le rejet populaire des lois du gouvernement Valls, depuis l'état d'urgence légal jusqu'au projet de déchéance de nationalité, pour trouver son climax contestataire dans la législation Macron/El Khomri, qui veut asseoir la victoire institutionnelle du capital sur le travail vivant. ${ }^{1}$ L'application de ce principe de flexicurité à la France est une tentative tardive d'épouser un schéma européen qui a imprimé sa marque à des degrés différents à l'Angleterre, à l'Europe de l'Est et du Sud. À Paris, le lien entre politiques nationales, directives européennes et lois de la mondialisation est aujourd'hui abordé sous la forme de questions irrésolues. Sommesnous entrés dans une modernité liquide, les grèves en masse seraient-elles une dernière révolte fordiste, s'agit-il de s'insérer dans le flux commercial du monde ou encore de s'organiser à travers un corporatisme concurrentiel, voire dans le repli souverainiste, en jetant par-dessus bord l'héritage internationaliste? A notre sens, la flexicurité illustre une nouvelle forme de contrainte, de domination et de soumission qui prend la forme paradoxale d'une bureaucratie liquide. Hiérarchique, implacable et autoritaire comme l'ancien talon de fer, mais insaisissable car irresponsable dans son principe même, qui est néanmoins un principe directeur et organisateur. La subordination salariale inscrite dans les contrats de travail, les conditions générales de vente contenues dans un contrat de service, les transactions bancaires et financières, les prêts du FMI ou de la Banque centrale européenne, les traités commerciaux internationaux, toutes ces formes sont tout à fait explicites et rigides lorsque leurs initiateurs imposent leur application. Cela se voit lorsqu'un particulier qui ne remplit pas les conditions est sanctionné, ou encore quand le gouvernement grec est mis au pied du mur par l'Eurogroupe en 2015. ${ }^{2}$ En cas de litige, les subalternes, salariés ou chercheurs d'emploi, souscripteurs de prêts immobiliers ou de crédits à la consommation, se voient aussi de fait privés des leviers institutionnels et juridiques qui ont plus ou moins marché dans le passé. ${ }^{3}$ Cela va d'un contrat de téléphonie mobile en passant par un licenciement abusif au remboursement de la dette par les Etats. Les accords transatlantiques esquissent dans leur version extrême la suppression de toute 
justice institutionnelle, au profit d'un droit privé d'arbitrage (Investor-state dispute settlement), de facto favorable aux grandes firmes. ${ }^{4}$

\section{La nouvelle loi de la mondialisation capitaliste}

2 La loi travail est le phénomène qui a révélé l'ampleur de ce problème politique aux yeux du grand public, puisqu'il est apparu dans le projet de loi qu'un patron pouvait décider d'imposer des licenciements sans motif sérieux ni recours, ou d'imposer le changement des contrats individuels contre les syndicats majoritaires. Il ne s'agit en rien d'un excès, d'un dysfonctionnement ou d'un dérèglement, mais bien au contraire du principe directeur de la flexicurité, en tant qu'expression d'une loi plus globale de la mondialisation capitaliste. L'origine historique, le cadre institutionnel, international et juridique de cette contre-révolution mondiale qui concerne aussi la France peut être clairement identifié. Le principe fut porté d'abord par l'exécutif des Etats Unis sous W. Clinton, qui a imposé au cours des années 1990 le conditionnement des allocations sociales à la reprise d'un emploi, le premier traité de libre échange des Amériques (Aléna / Nafta) en 1994, l'autorisation de règles opaques dans la gestion bancaire et financière, ainsi que l'alliance internationale des nouveaux démocrates avec la plupart des chefs d'Etat de la social-démocratie européenne. ${ }^{5} \mathrm{~A}$ commencer par A. Blair et G. Schröder, rejoints par F. Hollande. ${ }^{6}$ Cette stratégie s'est étendue à travers les organismes internationaux, l'OMC à partir de 1995, le FMI et les accords transatlantiques actuellement en négociation, qui visent l'imposition de nouvelles normes qui concernent le travail, la protection sociale, la santé et l'environnement. Le Parti socialiste français est en première ligne dans ce dispositif, ce dont témoigne la direction du FMI par D. Strauss Kahn jusqu'en 2011 et de l'OMC par P. Lamy jusqu'en 2013. L'origine politique et institutionnelle de l'imposition du nouveau principe en Europe est claire, elle date du sommet de Lisbonne auquel participe un ensemble de chefs d'Etat sociaux-démocrates européens dont L. Jospin, et qui fixe l'Agenda 2010. En découlent une série de directives cadres de l'Union européenne qui furent peu à peu imposées par les gouvernements des Etats membres en tenant compte des résistances et mouvements dans chaque pays (résistances parfois coordonnées sur le plan continental).

Dans la plupart des pays, cette même approche a provoqué de grands mouvements de contestation, en particulier en Allemagne (2004), en Espagne (2009-11), en Grèce (2010-16), en Italie et au Portugal, mouvements qui se sont produits de manière décalée et avec des effets variés. Dès le début, les principaux perdantes et perdants de cette politique furent les prolétaires les moins qualifiés, les femmes salariées et les jeunes. Le programme de la flexicurité et sa dynamique se heurte à des résistances et oppositions majeures en Europe qui touchent aujourd'hui la France. La Confédération européenne des syndicats verse désormais dans l'opposition, en bloc, elle enregistre un changement de tendance majeur. En Europe du Sud, le retournement est explicite et massif, porté par de grands mouvements depuis 2011, suivis de renversements politiques ou de crises gouvernementales en Grèce, au Portugal, en Espagne et sous d'autres formes en Italie. La France est en train de suivre ce mouvement inégal mais combiné, à travers l'opposition à la loi travail en 2016, après l'avertissement que fut le retrait du Contrat première embauche en 2006. Bien entendu, la mise entre parenthèses du programme de la flexicurité, en France, entre la fin des grèves de 1995 et le début de la mise en place 
de l'Euro en 2000 n'était due qu'à la forte impression contestataire de cette période, tout comme le renversement actuel dans le sud de l'Europe se fonde sur des mouvements de masse et des espaces publics oppositionnels de grande échelle. En Grande Bretagne, l'opposition syndicale et travailliste a clairement tourné le dos à la stratégie flexicuritaire, identifiée aux années Blair, au moment d'une primaire qui a vu l'élection sans appel de l'opposant historique J. Corbyn en 2015-16 avec le soutien des principaux syndicats. L'Europe de L'Est a basculé dans une opposition anti-libérale de type nationaliste, anti-communiste et autoritaire, à l'image de l'Hongrie et de la Pologne. L'Allemagne et les Bénélux sont travaillés par des contradictions significatives, des mouvements critiques s'y expriment à l'encontre des partis socialistes qui y co-gouvernent. ${ }^{7}$

4 Le mouvement oppositionnel à l'encontre la loi El Khomri, qui a associé plusieurs millions de citoyenn(e)s, ne se limite plus à la définition d'un mouvement social, en opposition à la politique décisionnaire, sauf pour les commentateurs qui restent attachés à ce terme, défendu par Alain Touraine et la gauche rocardienne depuis l'échec stratégique du mouvement ouvrier d'Europe occidentale depuis $1978 .{ }^{8} \mathrm{Le}$ mouvement de 2016 se distingue par son opposition directement politique, qui s'affronte à l'Etat, comme en témoigne le recours à la constitution autoritaire qui permet de supprimer le vote parlementaire et la négociation syndicale, les violences policières récurrentes, l'usage de l'état d'urgence pour interdire ou restreindre le droit de manifester. Ce fait est mis en musique par le verbe vindicatif de l'exécutif, comparant la CGT au Front national, les militants syndicaux et associatifs à des casseurs et terroristes potentiels, l'opposition à une horde. Les répercussions directement politiques du mouvement oppositionnel du printemps-été 2016 sont visibles : éviction de l'exécutif Hollande et Valls, discrédit abyssal de leur programme et bilan dont témoigne le vote des "primaires citoyennes" en février 2017, renversement de l'équilibre des forces syndicales en faveur d'une majorité oppositionnelle, radicalisation de la jeunesse scolarisée ou salariée, de l'espace militant, ouverture d'un vaste espace idéologique à gauche du Parti socialiste et du gouvernement qui se manifeste aussi sur le plan électoral, vitalité des essais et revues d'intellectuels ou critiques de gauche et accroissement du public qu'ils rencontrent. ${ }^{10}$

5 Les quatre listes de gauche, opposées à l'exécutif Hollande et en faveur de l'abrogation de la loi travail obtiennent près de $28 \%$ au premier tour, soit 9.978128 voix. Selon les premières enquêtes de sociologie électorale, un tiers des ouvriers et employés a voté pour cette position de gauche, tandis que le FN décline ici d'environ 10 points pour arriver désormais environ à la même proportion. ${ }^{11}$ Aussi, selon une étude de l'IFOP de 2017, 60\% des ouvriers déclarent n'avoir "jamais voté pour le FN". Le FN n'est pas le parti du vote ouvrier, et n'a jamais été un parti ouvrier.

6 Malgré l'opposition intellectuelle, sociale et politique des courants de gauche, la flexicurité est encore supportée, soutenue ou tolérée en France par de larges pans de l'électorat socialiste, ce qui permet à $\mathrm{E}$. Macron de maintenir ce projet, relayée par les droites politiques, par des organisations syndicales minoritaires mais significatives (à l'instar de la direction de la (fdt), des intellectuels et universitaires à la retraite mais très médiatisés (Anthony Giddens, Jürgen Habermas, Jacques Attali). Certains théoriciens de la flexicurité ont récemment changé de cap, dont Wolfgang Streeck, l'un des théoriciens du corporatisme concurrentiel de l'ère Schröder, et le regretté Ulrich Beck. ${ }^{12}$ D'autres ont maintenu leur position, à l'instar de Jürgen Habermas qui fut le 
président du club européen de Dominique Strauss Kahn dans les années 2000 avant de soutenir Emmanuel Macron, ou de Pierre Rosanvallon qui fut le fer de lance d'une pétition pour les réformes d'Alain Juppé et contre le mouvement de grève en $1995^{13}$. Rosanvallon, un temps co-animateur de la fondation Saint Simon aux côtés d'Alain Minc, a reformulé son argumentaire pour reprendre en compte le conflit social, sans pourtant rien céder sur sa position de fond de $1995 .{ }^{14}$

Ce média ne peut être affiché ici. Veuillez vous reporter à l'édition en ligne http://

E. Macron et son soutien déclaré, J. Habermas, en avril 2017 à Berlin. Droits réservés.

8 La critique et la contestation récente de la "loi travail" a surtout été abordée, de manière assez traditionnellement syndicale, comme un moyen d'affaiblir le code du travail, les conventions collectives et de baisser les salaires, alors que sa dimension directement politique va au-delà de ces aspects circonscrits, elle réside dans l'imposition d'une nouvelle loi de la mondialisation capitaliste. Cette dimension politique du droit apparaît dans le terme même de flexicurité qui définit la loi travail et qui se prolonge dans le vocabulaire de l'inversion de la hiérarchie des normes. Cela trahit une motivation intrinsèquement politique dans un langage cachant mal l'enjeu du pouvoir : qui décide? Il s'agit de rendre malléable la force de travail en garantissant une sécurité juridique au patronat et aux entrepreneurs, par la force de loi. C'est précisément l'objet de la législation que Karl Marx évoque à propos de l'accumulation du capital dans l'Angleterre capitaliste depuis le 15ème siècle, où des lois successives ont permis l'expropriation des communautés paysannes en faveur des grands éleveurs de mouton, venus nourrir l'industrie textile, complétées par d'autres lois pour contraindre les anciens paysans, déracinés et errants, d'accepter un travail salarié dans les grandes villes s'ils ne voulaient pas être brûlés au fer pour vagabondage, enfermés et mis au ban de la société. ${ }^{15} \mathrm{Au}$ 19ème siècle, la législation du travail garantit d'abord des conditions d'exploitation stables, avant de concéder la limitation des heures de travail, sous l'impact du mouvement ouvrier, limitation que Marx appelle la victoire du travail vivant sur l'économie du capital. Pareille interprétation fut rejetée par les philosophes libéraux, les économistes bourgeois et les sociologues de l'époque, Emile Durkheim en tête. Aujourd'hui, la compréhension de la mondialisation capitaliste, avancée par Marx dès le milieu du 19ème siècle, est entrée dans l'état de l'art des sciences humaines, pour être aussitôt relativisée dans sa portée critique par A. Giddens, W. Streeck, J. Habermas, A. Touraine, M. Wieviorka et A. Honneth au sein de la socialdémocratie européenne. ${ }^{16}$

Zygmunt Baumann a parlé, dès 2000, d'une modernité liquide, qui serait marquée par le désengagement et affaiblissement des liens mutuels qui unissaient jadis le travail et le capital, dans un capitalisme qui serait devenu "léger et librement flottant". ${ }^{17}$ La même idée concerne l'Etat nation obsolète au milieu de ce flux financier, extra-territorial, communicationnel, où ce n'est plus le travail en actes, mais l'apport d'idées qui engendre les profits. Le sociologue admet que la liberté du capital n'est pas encore devenue totale, que des appuis locaux existent et que des gouvernements peuvent ici et là entraver le mouvement capitaliste, mais cette réserve vient surtout souligner la pleine puissance et la validité globale de la thèse. Elle contourne une idée centrale de Karl Marx, qui a montré que le capital lui-même se compose d'une partie fixe 
(investissements, matériaux, moyens de production) et d'une partie variable (les salaires). En fait, la diminution de la part variable a toujours fait baisser les profits, hier et aujourd'hui. Même si de grandes entreprises font monter leur cours en bourse à court terme à travers des plans de licenciements, elles perdent à plus long terme, sauf si elles captent le travail de sous-traitants, de main d'œuvre à bas prix dans des pays du Sud, etc. La spéculation boursière et financière n'a jamais garanti une rentabilité durable, comme la grande crise de 2008 l'a montrée. Chez Marx, le travail vivant des salariés apporte plus value, idées, intelligence collective et coopération qui permettent la valorisation du capital. Postuler que le capital et le travail seraient en train de se défaire est une pure spéculation philosophique. Le titre du présent article, $L a$ bureaucratie liquide, est volontairement paradoxal et ironique, même s'il doit être lu comme un hommage indirect à Baumann qui a le grand mérite d'avoir analysé les conditions organisationnelles de la destruction des juifs d'Europe de l'Est sous le nazisme, et d'avoir soutenu l'opposition morale à la politique militariste, liberticide et anti-sociale de l'ancien Premier Ministre britannique, Sir Anthony Blair, la personnification du blairisme. La distance critique à l'égard des propositions postmodernes qui pensent que les catégories et concepts les plus lourds de la modernité depuis la révolution française se seraient dissipés - le travail, le capital, l'Etat, l'idéologie - nous vient de la Théorie critique dans une filiation qui va de Theodor Adorno et Walter Benjamin à Jean-Marie Vincent et Oskar Negt, en passant par le féminisme de Nancy Fraser et Regina Becker Schmidt. ${ }^{18}$ Est-ce qu'une société qui tolère l'état d'urgence issu des anciennes guerres coloniales, et dont l'exécutif décline autant le discours national que celui de la valeur travail, au point d'indexer son propre avenir sur l'inversion de la courbe des statistiques du chômage, peut encore sérieusement être caractérisée comme postmoderne? ${ }^{19}$

L'idée que la circulation fluide des capitaux financiers signerait une modernité liquide va aussi à l'encontre d'un constat fondamental de la sociologie de Max Weber, qui a montré que la première bureaucratie moderne est née de la comptabilité bancaire, du système financier, puis de la gestion des flux et carnets de commande des grandes entreprises, en Europe. ${ }^{20}$ La Banque centrale européenne, basée à Francfort-sur-leMain, est le sommet de cette évolution historique, elle ne tolère aucun écart à la norme monétaire. Aujourd'hui, les flux monétaires, financiers ou boursiers sont gérés par d'énormes agences, machines et logiciels, organisés par des armées de managers, de traders, informaticiens et employés. Le management de la Société générale, localisée à Paris, aurait aimé faire croire qu'un seul Trader (J.Kerviel) puisse déplacer d'énormes flux financiers sans contrôle direct ni gestion précise, mais la Justice a démontré le contraire. Les flux financiers, les flux numériques, les procédures des services, les échanges mondiaux, tous ces flux créent d'immenses besoins de gestion et de contrôle qui sont aux fondements sociologiques de la bureaucratie liquide. Marx avait pressenti le phénomène de bureaucratisation de la social-démocratie, puis Weber a poursuivi l'analyse de la bureaucratie des entreprises, des banques, des administrations et des partis de masse. Cette forme bureaucratique fut résumée sous le terme fordisme par A. Gramsci, puis par des sociologues et économistes, après la seconde guerre mondiale. Le cas de figure du chef d'industrie américain Ford qui a donné le nom au fordisme illustre bien le passage de la bureaucratie industrielle à la bureaucratie liquide. Henry Ford personnifie la production et la consommation de masse, soutenu par un étroit contrôle gestionnaire des horaires, des gestes de travail, des salaires et des prix, une violente répression syndicale interne et une répression armée à l'extérieure des usines grâce à 
l'Etat, doublé d'un discours idéologique stéréotypé. Ford est l'auteur de traités antisémites mémorables et fut décoré de son vivant par le 3ème Reich. ${ }^{21}$ Plus tard, Ford s'opposa au New Deal. Cela vient rappeler le rôle de la domination directe dans le fordisme qui n'est pas conditionné à un développement démocratique, comme le montre aussi l'origine de l'entreprise Volkswagen dans le complexe militaro-industriel du national-socialisme. Aujourd'hui, l'industrie automobile reste un secteur central sur le marché mondial, mais la domination bureaucratique s'est déplacée. La bureaucratie actuelle peut toujours activer la contrainte en cas de conflit direct, comme le montre le cas Mikkael Wamen, syndicaliste de Goodyear condamné à une peine de prison avec sursis en 2016-17, mais la bureaucratie liquide active d'autres ressorts majeurs. Elle n'expose pas ses dirigeants ou managers, mais avance des critères chiffrés comparés pour impliquer les salariés dans la gestion concurrentielle (affichage comparé de la production et de la rentabilité sur les lieux de travail, évaluations individuelles). L'invocation du marché et de ses nombres tend à remplacer l'intervention directe et autoritaire du patron et du contremaître. De même, la répression ouvrière, interne et publique, tend à être subjuguée par la soumission volontaire des salariés, par le truchement de la psychologie. La peur du licenciement, du chômage comme mort sociale et le besoin constant d'une identification positive des individus conduit à l'acceptation passive ou explicite de la dégradation des conditions de travail et de rémunération. Ce genre de gestion amoindrit les résistances classiques, par exemple l'absentéisme et la grève du zèle ou la grève sauvage, mais ne les supprime pas et d'autres formes de résistance prennent le pas, dont l'arrêt maladie et l'apparition involontaire de maladies psychiques au travail pouvant aller jusqu'au suicide. Une conduite conformiste devient la norme implicite et non plus explicite dans le monde du travail; des accords d'entreprise sont soumis au vote dans le but d'obtenir des adhésions plébiscitaires. Cela concerne tous les secteurs d'activité, de l'industrie au commerce en passant par la santé. Le chiffrage comparé de la compétitivité fait l'objet d'une gestion bureaucratique individuelle, collective et managériale à tous les niveaux, grâce à des logiciels et supports numériques, dans la gestion des ressources humaines comme dans le suivi des activités. L'un des exemples du contrôle numérique est le scan, qui sert à contrôler les ventes dans les supermarchés, les stocks, les commandes et flux logistiques et commerciaux, de la logistique à la poste et aux transports, en passant par chaque poste de travail. Les salarié(e)s sont contrôlés et disciplinés par ce type de suivi numérique et individualisé. Le flic est dans le flux. Le calcul serré, à travers le scan, des espace-temps entre chaque acte de travail ou de vente n'est rien d'autre que le traditionnel calcul taylorien du temps et des horaires dans les conditions $\mathrm{du}$ numérique, à un niveau global de la bureaucratie liquide. Ce principe prétendument post-fordiste de chiffrage comparé s'est étendu à l'évaluation de toutes les activités économiques et sociales, dont la fonction publique et l'éducation, puis à la gestion institutionnelle au sein de l'Union européenne. Le meilleur exemple est la norme de déficit budgétaire de 3\% des Etats membres de l'UE qui fut fixée de manière arbitraire dans les années 1990 mais qui s'est érigée en seuil politique effectif.

11 Le sommet européen de Lisbonne de 2000 a inauguré une méthode politique qui cherche à compenser les lacunes de la subsidiarité, en décidant de traiter les problèmes thématiques et les conflits intergouvernementaux des pays membres selon un schéma managérial connu de la sociologie des organisations, en fixant par avance des procédures et des étapes dans le cadre d'un programme de dix ans, l'Agenda 2010. Il s'agit d'une sorte de management par projet inspiré des grandes entreprises, appliqué à 
la construction européenne, selon la MOC, la " méthode ouverte de coordination »"2 . La MOC fonctionne selon le principe de gestion du «benchmarking» international, consistant à identifier les «meilleures pratiques » et les points faibles des dispositifs nationaux respectifs, à partir d'une grille d'analyse pré-établie qui mesure les performances et contre-performances de manière quantitative. Les défauts constatés à travers le MOC doivent être améliorés par des plans d'action chiffrés comportant des délais précis. Pareille logique se décline ensuite sur le plan des Ministères, des Régions, etc. Les chiffres ainsi obtenus sont désormais traduits en coûts à travers un logiciel économique, le SCM (Standard Coast Model) ${ }^{23}$ que nous avons expérimenté à l'occasion d'une étude pour la Commission européenne au sujet de l'évaluation de l'usage flexible du temps de travail ${ }^{24}$. Le SCM prétend calculer toute sorte de politique publique en termes de coût économique, son ambition est donc de quantifier toute action en termes monétaires, sans égard des particularités historiques, sociales, régionales ou de genre. Bien entendu, la privatisation des Job centers (gestion du chômage) dans la plupart des pays européens, ou les partenariats publics-privés dans l'éducation ou la santé, ont crée des besoins de gestion accrus, autrefois assurés par les institutions étatiques, et conduisent à un management aussi opaque qu'instable qui se sert de critères quantitatifs pour masquer son désarroi.

L'association de la domination bureaucratique, managériale et institutionnelle, et de la gestion néocapitaliste des flux n'est pas une nouveauté absolue. L'alliance de l'Etat et de la concurrence marchande l'est encore moins, elle fut portée par Ford et, auparavant, par le second empire sous Napoléon III à partir de 1850. La France illustre, depuis des années, l'association d'une politique à la fois libérale et étatique autoritaire, prête à suspendre la convention européenne des droits de l'homme pour appliquer la déchéance de nationalité. Cette politique étatique n'a rien de postmoderne, elle résume tous les aspects problématiques de la modernité étatique, y compris son versant bonapartiste. Christian Laval a apporté, en France, une meilleure compréhension de l'ordo-libéralisme allemand, cet alliage d'un libéralisme économique structuré par des règles légales et conventionnelles, qui diffère du premier libéralisme anglais par une mobilisation continue de l'Etat, assorti de l'implication des organisations syndicales et patronales. ${ }^{25}$ L'Etat fait ici partie de la discussion critique, au lieu de se trouver idéalisé comme une solution en soi. Le principe Macron cherche explicitement à imiter ce modèle, dans les discours, la législation et la pratique des accords d'entreprise. Pareille réception de l'ordo-libéralisme passe cependant à côté de la singularité historique et constitutionnelle de l'Allemagne, qui remonte à la révolution des conseils de novembre 1918 et la proclamation de la république de Weimar qui constitue toujours le socle formel de la république fédérale actuelle, malgré l'expérience du nazisme ou encore de la république socialiste est-allemande après la guerre. Le mouvement conseilliste originaire a continué d'agir sous les conditions de l'économie de marché, à travers un mouvement syndical unifié et puissant, qui a directement influencé la législation et le droit du travail, comme Jean-Marie Vincent et Peter von Oertzen l'ont montré dès 1963, rejoint plus tard par des chercheurs franco-allemands autour de Pierre Bourdieu dont nous avons publié les débats en $1998 .{ }^{26}$ L'ordo-libéralisme, qui correspond à une théorie juridique et ne fournit donc aucune analyse pratique des institutions et organisations nationales, a tenu compte et intégré ces mouvements et conditions historiques. Le projet européen de la flexicurité cherche précisément à contourner ces résistances et singularités historiques, qui n'apparaissent qu'indirectement à travers l'Etat national, à 
travers une forme de concurrence et de coopération contrainte, qui concerne aussi directement la France.

L'accent qu'y mettent la plupart des critiques de gauche actuels sur l'aspect anti-social, inégalitaire, précarisant ou appauvrissant la loi travail, qui focalise le regard sur des symptômes, évite de prendre directement en considération cette dimension légale, historique et politique, qui nous apparait pourtant claire si nous portons l'analyse à l'échelle internationale et non pas franco-française. L'affaiblissement historique du mouvement ouvrier traditionnel date de 1978, bien avant la chute de l'Union soviétique en 1991 qui symbolise l'entrée dans la phase actuelle de la mondialisation capitaliste, a laissé la voie ouverte à une actualisation d'une législation du travail qui consiste à contraindre le travail plutôt qu'à garantir un droit collectif des salariés. C'est précisément le principe de la flexicurité qui fut portée dès les années 1990 par "l'internationale socialiste" et démocrate qui a joué un rôle clé dans cette soumission juridique, légale et idéologique du travail vivant au capital, en assurant la collaboration d'une importante partie du salariat organisé, dont les ouvriers qualifiés et organisés de l'industrie automobile et chimique, par exemple. Sans cette collaboration, la nouvelle législation flexicuritaire aurait été perçue comme une simple agression patronale et réactionnaire. Le contenu contraignant et régressif de la nouvelle loi, que nous allons présenter comme la loi de la mondialisation capitaliste, a néanmoins fini par percer aux yeux des citoyens concernés par le dispositif, dont les ouvriers et employées (en majorité des femmes), les salariés précaires, allocataires et demandeurs d'emploi, ainsi que les jeunes en formation ou en étude qui ont saisi, en masse, les conditions que cette législation leur réservait, s'apparentant in fine comme une forme d'esclavage salarial (Marx). Les secteurs les plus protégés et le mieux syndiqués ont fini par basculer dans l'opposition, à l'instar des syndicats de la métallurgie et de fonctionnaires allemands, les syndicats de cadres français, ou plus récemment les Dockers qui commencent à se définir comme une partie intégrante d'un mouvement global. ${ }^{27} \mathrm{Au}$ bout du processus, cela signifie des salaires horaires à un Euro brut (en Allemagne par exemple), des contrats de travail à zéro heures de travail garanties par mois comportant toutes les obligations légales pour le salarié signataire, la privation totale de droits pour certains demandeurs d'emploi, l'imposition de stages non rémunérés ou de formations dépourvues de toute qualification (par exemple, "jouer" à la caissière de supermarché avec de la monnaie en plastique, formation dispensée par des centres pour l'emploi allemands) et plus globalement, la transformation de droits constitutionnels en mesures individuelles à la discrétion des administrations spécialisées de l'Etat (aide sociale ou familiale; Job centers; formation ou études).

Notre approche critique n'est pas exclusivement conceptuelle même si nous y avons consacré plusieurs livres ${ }^{28}$; elle se nourrit d'un même mouvement d'une multitude de recherches empiriques que nous avons conduit depuis 1999 sur toutes les facettes la flexicurité en Europe : transformations du travail en entreprise, de la recherche et développement privé, réforme des agences pour l'emploi et des politiques publiques, projets de formation et de l'insertion professionnelle, précarisation, travail transfrontalier. J'ai mené ces enquêtes dans le labyrinthe du Minotaure, pour le compte d'entreprises multinationales, pour des ministères allemands, pour la Banque mondiale et la Commission européenne, en acceptant plutôt de ne jamais obtenir l'autorisation de publier les rapports qui ont sont issus plutôt que de dévier d'un pouce des conclusions critiques que j'ai formulé. Dans le passé, j'ai bénéficié de mon statut de chargé de recherche pour des Universités ou Instituts de recherche, afin de présenter 
mes propres vues à travers des articles scientifiques qui ont échappé à la censure des administrations étatiques et internationales. Désormais, il est temps de livrer un point de vue plus global, un peu dans le style de Cornelius Castoriadis qui passa du rôle d'économiste officiel de l'OCDE et de sa critique semi-clandestine publiée sous pseudonyme, à l'exposé d'une critique radicale du "bureaucratico-capitalisme" en son nom propre..$^{29} C^{\prime}$ est la raison pour laquelle je vais exposer les chantiers pratiques du droit du travail et de la flexicurité au fil des pages qui suivent. David Graeber montre pour sa part à quel point la valorisation capitaliste et le travail salarié s'inscrivent, aujourd'hui plus que jamais, dans des dispositifs bureaucratiques lourds qui se situent à l'opposé d'un capitalisme léger et sans attaches..$^{30}$ Nous avons signalé à quel point le travail s'agrège encore selon la distribution territoriale des capitaux en Europe, à travers une étude des créations d'emploi régionales suite à la crise économique de 2008. ${ }^{31}$ La fuite des capitaux, qui est l'une des raisons de l'effondrement grec, prouve encore l'importance de leur implantation locale. Aussi, la crise mondiale actuelle n'est pas l'effet d'une sphère financière extra-territoriale, sinon extraterrestre, mais a pris son essor aux Etats Unis, dans la spéculation sur les dettes immobilières. La crise des subprimes fut déclenchée par une surévaluation de biens immobiliers, localisés dans des villes US, et l'incapacité des salariés endettés de rembourser les traites. Le capital dépendait donc en dernier recours de valeurs immobilières, d'habitants et de salariés localisés dans des endroits précis. S'il est vrai que la domination bureaucraticocapitaliste et la soumission salariale ont changé de forme, elles ne se liquéfient pas, mais s'affirment souvent en pire, selon des modalités anciennes connues depuis le 18ème siècle : harcèlement, travail précaire, saisonnier, contraint, clandestin, et dépendance directe sont au menu. Lorsqu'elle est tolérée, la domination et soumission nouvelle parvient à se mettre en retrait et à s'organiser de manière indirecte, ramifiée, labyrinthique et dans l'évitement d'une prise de responsabilité explicite, au nom de principes supérieurs (les marchés, la nation, les jeunes générations, l'avenir de l'Europe).

La flexicurité est une manifestation typique de cette configuration, elle résume le projet phare de l'Union européenne depuis l'an 2000, qui porte enfin un nom en France : Emmanuel Macron. E.M. s'affiche comme une position centrale, comme une personnification idéale du mainstream international. Peu importe le nom ou le label, le champ de bataille de la flexicurité qui traverse l'Europe entière depuis près de vingt ans porte toujours sur les mêmes sujets. Il s'agit de soumettre les salariés aux directions des entreprises, les précaires aux aléas du marché du travail, les demandeurs d'emploi et allocataires aux injonctions des agences qui les gèrent et les écoles ou universités au maitre-mot de la valorisation marchande des compétences. La subordination des salariés aux employeurs, aux chefs et à la direction des ressources humaines doit s'accroître, la domination de la bureaucratie s'étendre. Le temps de travail, la mobilité des salariés doit s'étendre au maximum. Karl Marx a remarqué que la limite absolue de la journée de travail était de 24 heures, mais la flexibilité capitaliste actuelle va plus loin, en gérant le temps des salariés sur l'année, voir sur plusieurs années, sinon sur toute la vie. Les salaires et les allocations sociales inconditionnelles doivent baisser, continuellement, au nom de la concurrence internationale, de la compétition de tous contre tous, bien organisée cependant afin de ne pas déstabiliser le système global. Tout ce qui gêne et contrarie ce mouvement doit périr, à commencer par la gauche historique, l'idée même de la lutte des classes et de la résistance, sinon le simple concept de critique sociale ou de toute visée utopique. En Allemagne, la cour 
constitutionnelle a reconnu que les lois Hartz qui ont transposé la flexicurité violent le principe constitutionnel car les prestations prévues ne garantissent pas la dignité humaine ${ }^{32}$ Loin de la rhétorique de l'entrepreneur ou du modernisateur, l'enjeu de la flexicurité se situe bien au niveau de la loi fondamentale, de la constitution qui fonde la société, de son principe constitutif. L'obstination avec laquelle la stratégie européenne de Lisbonne est poursuivie par les droites et la social-démocratie européenne, jusqu'à sa propre perte, peut donc étonner, si on la considère comme un simple compromis continental. En réalité, il s'agit de l'application stratégique d'un projet plus global au sein du capitalisme mondial.

16 A l'origine, le terme de flexicurité a officiellement éclos lors d'un sommet européen au Portugal. L'approche fut baptisée la stratégie de Lisbonne par les chefs d'Etat et de gouvernement lors du sommet européen de 2001 qui s'est tenu dans la capitale portugaise. Elle devait permettre, en l'espace de dix ans, de faire de l'Europe la première économie du monde, basée sur le savoir, de rétablir le plein emploi et de diminuer toutes les inégalités, régionales, sociales, générationnelles et sexuées. L'argot officiel de l'Union européenne parlait donc de l'Agenda 2010, ce qui est le terme exact qui fut repris par le Chancelier Schröder pour donner un nom à une batterie de lois, de mesures et de conjectures qu'incarne aujourd'hui M. Macron. Sur le plan de l'Union européenne, le cycle qui va de l'essor à l'échec de cet agenda coïncide avec la présidence Barroso à la tête de la Commission (2004-2014), mais ses grands objectifs sont maintenus par son successeur Juncker. Les objectifs de la stratégie de Lisbonne furent tous ratés, sans exception aucune, comme les bilans officiels de la Commission européenne l'ont reconnu officiellement dès $2010 .{ }^{33}$ Son projet devait faire de l'Europe la zone la plus prospère du monde, grâce son rôle de pointe dans l'économie du savoir. Le virage vers le capitalisme cognitif, vers les services immatériels au détriment des industries anciennes et nouvelles, devait permettre le plein-emploi, la résorption des inégalités régionales et sociales, liées au genre, au niveau de qualification et à l'âge. Au moment du bilan des dix premières années de cette stratégie, l'Europe a connu la récession puis la stagnation économique, un chômage de masse dont la comptabilité la plus restrictive a dépassé les $12 \%$ en moyenne en 2014 , et les $40 \%$ parmi les jeunes en Grèce, au Portugal et en Espagne. Selon le calendrier de Lisbonne, le plein emploi aurait dû être réalisé dès 2010. Les inégalités ont explosé, notamment entre jeunes et seniors, entre salariés fortement et peu qualifiés, entre hommes et femmes, alors que les écarts de revenu se creusent et les disparités régionales entre Nord et Sud menacent d'effondrement l'édifice européen. L'Europe était appelée à devenir la première économie du savoir de la planète, mais le projet est resté à l'état conceptuel, assez bien représenté par la théorisation du capitalisme cognitif par Yann Moulier-Boutang. ${ }^{34}$ Aujourd'hui, la France et l'Europe du Sud tentent d'endiguer la désindustrialisation et les jeunes diplômés n'y entrevoient aucune perspective durable si ce n'est l'émigration.

En Allemagne, le principe de la flexicurité est personnifié par Peter Hartz, l'expert le plus exposé du gouvernement Schröder (1998-2005). Au printemps 2014, le président Hollande a reçu Monsieur Hartz comme visiteur à l'Elysée, avant d'appliquer ses principes qui sont devenus globaux. Il s'agit du concepteur des "réformes structurelles" qui furent appliquées en Allemagne il y a dix ans, à travers quatre grandes lois qui portent désormais son nom. Tous les leitmotivs du débat public hexagonal actuel sont inscrits dans cette législation : dérèglementation du marché du travail, flexicurité, activation et contrôle des chômeurs. L'instauration d'une sorte de RSA, à travers la quatrième loi Hartz, fait qu'en allemand courant ou familier, l'on dit "Hartz 4" pour 
nommer cette forme d'allocation. M. Hartz est devenu le symbole et l'incarnation d'une approche qui se veut à la fois une construction théorique, idéologique, pratique et politique. Peter Hartz a publié un livre dans lequel il conçoit une culture politique centrée sur l'autogestion individuelle, où il va jusqu'à envisager un "homme nouveau". $\mathrm{Au}$ zénith de sa carrière il a obtenu, en 2004, le titre honorifique de professeur de l'institut universitaire technique de l'Etat de la Sarre, région dont il est originaire, grâce à son expertise en tant que DRH et d'un diplôme de gestion obtenu au sein de ce même IUT, sans avoir produit de thèse universitaire. Responsables politiques, syndicaux et patronaux, entrepreneurs, élus, sociologues, experts, journalistes, chefs de gouvernement et représentants de la Commission européenne ont contribué à donner corps à la conception dont il est question, dès les années 1990. M. Hartz en personne a successivement joué la plupart de ces rôles, depuis sa carrière en tant que directeur des ressources humaines chez Volkswagen, aujourd'hui la plus grande entreprise mondiale de l'automobile, en passant par les commissions d'experts, les relations publiques et finalement la fonction du premier conseiller du gouvernement fédéral allemand.

En France s'affiche une position hagiographique qui défend le principe Hartz contre toute sorte de critique. Elle prend souvent l'apparence de l'expertise professionnelle ou scientifique. Cette posture s'illustre par un récent article d'Isabelle Bourgeois qui promet de rétablir la vérité à l'encontre des caricatures, amalgames et distorsions populistes que les opposants à la réforme auraient introduits dans le débat francoallemand ${ }^{35}$ La voie serait "largement ouverte à l'amalgame", car "même les observateurs les plus irréprochables s'alimentent souvent sans le savoir à des sources allemandes situées dans le camp des opposants à ces réformes". Face à de telles " manipulations multiples des réalités", il s'agit de "dresser un état des lieux factuel des réformes apportées par les lois Hartz comme des polémiques allemandes sur la "flexicurité " afin d'apporter quelques informations objectives indispensables au débat français". L'article, qui est extrêmement détaillé, voudrait se distinguer par une approche factuelle, objective et scientifique. Isabelle Bourgeois omet de préciser que l'institut au nom duquel elle publie ce texte, le CIRAC, est un partenaire officiel de l'équivalent de Pôle emploi en Allemagne, la Bundesagentur für Arbeit, donc d'un organisme qui fut crée pour appliquer les réformes Hartz. Dans son article pour le bulletin du CIRAC, Isabelle Bourgeois commence par déclarer que le gain de compétitivité induit par les réformes Hartz serait "incontestable", en citant une seule source, à savoir un autre article publié dans le même bulletin du CIRAC, sous la plume de Bert Rürup. Cet auteur ne s'est pas distingué en tant que chercheur, mais comme conseiller du gouvernement Schröder au moment où furent adoptées les réformes Hartz. La preuve incontestable et factuelle qu'Hartz avait raison serait donc que les organismes et experts qui ont travaillé avec lui le disent. Bourgeois fait ensuite part de son regret politique que l'ensemble des partis de la gauche parlementaire et du syndicalisme allemand auraient succombé à la démagogie des opposants, qu'elle assimile à la totalité de la gauche parlementaire et aux principaux syndicats. Elle étaye pareille thèse par un seul document, un "tract commun" d'ATTAC et du syndicat allemand Verdi. La posture scientifique et objective se dissipe ainsi rapidement face au positionnement doctrinaire des apologues du principe Hartz, dénuée de toute démonstration empirique.

Le discours de la flexicurité de type Hartz ou Macron participe bien du nouvel esprit du capitalisme, pour une large part fantasmagorique, que Boltanski et Chiappello ont mis en évidence à partir des manuels de management des années $1990 .{ }^{36}$ Cependant, cette analyse des textes managériaux ne prétend pas et ne parvient pas à décrire ce qui se 
passe au moment où la flexicurité veut imposer sa nouvelle vision à des salariés récalcitrants, tout comme les formes anciennes d'esprit du capitalisme se sont heurtés à de multiples résistances qui vont de l'absentéisme à la grève sauvage. Lorsque la transformation se passe relativement bien du point de vue de la flexicurité, sous l'effet de la peur du déclassement sociale, ou de l'enthousiasme fantasmagorique des salariés, comme lors de la première phase du "modèle Volkswagen" sous Hartz (1994-2004), l'opinion publique et même les sociologues peuvent partager l'impression que le discours managérial et l'attitude des salariés concordent plus ou moins. Pourtant, dans la plupart des cas, le principe de la flexicurité ne fait que déplacer des lignes de conflit anciennes, dans l'industrie automobile et ailleurs, ce que prouva les grèves dures qui ont suivies dans plusieurs sites de production. Des exemples que nous avons suivi de près sont les usines Opel à Bochum, le site de développement de logiciels d'IBM à Düsseldorf, mais il est possible de songer à Renault en France ou bien d'autres cas. A côté de ces formes presque classiques, l'extension des maladies professionnelles de type psychique, dont tous les phénomènes de dépression, ont actualisé la résistance passive plus ancienne.

La transformation du discours managérial du DRH Hartz en batterie législative a ensuite provoqué des oppositions culturelles, sociales plus que syndicales et publiques d'une ampleur inégalée depuis la chute du mur de Berlin, qui ont plus tard abouti à la désagrégation du gouvernement Schröder, en 2005. Avant même l'entrée en vigueur des lois Hartz, leur principe avait provoqué des grèves sauvages aux usines Opel de Bochum et le déferlement d'un quart de millions de citoyens dans la rue dans une centaine de villes, en 2004, sans aucun appel des grandes organisations syndicales. ${ }^{37}$ Cette expérience sociale est à la base du basculement des principaux syndicats des services et de l'industrie, Ver.di et IG Metall, dans le camp de l'opposition au principe Hartz. L'onde de choc s'est prolongée à travers l'espace public, à travers le recul durable du parti social-démocrate à $25 \%$ des voix, face à la progression des gauches anticapitalistes et écologistes opposées à divers degrés au principe Hartz. Le principal quotidien - la Frankfurter Allgemeine Zeitung - qui avait relayé les propositions hartziennes et dont la maison d'édition a publié son livre Job-Revolution, a suivi le retournement global de l'opinion publique en accordant des séries d'articles spéciales à la critique du capitalisme tardif.

21 Soyons précis sur le principe politique et moral qu'incarne P. Hartz, ancien DRH de VW qui est un repris de Justice. Dans les années 2000, les signataires des accords VW, M. Hartz et $\mathrm{M}$. Volkert furent condamnés à des peines de prison pour détournement de fonds et pour corruption en tant que mandataires au sein de l'entreprise VW. M. Hartz a publiquement avoué sa culpabilité concernant les 44 chefs d'accusation devant le tribunal de Braunschweig. ${ }^{38}$ Il s'avère lors du procès que le responsable de la DRH a favorisé l'accord avec certains représentants du personnel en les invitant, aux frais de l'entreprise, à des voyages gratuits en Thaillande, parfois accompagnés de la visite de bordels locaux, selon l'acte d'accusation. M. Volkert fut condamné en première et deuxième instance en 2007. Ces pratiques managériales discréditent le dispositif imaginé par M. Hartz, présenté comme une incarnation du management participatif, de la gestion éthique d'une multinationale, donc du nouvel esprit du capitalisme. Aussi, la cogestion élargie au sein de VW apparaît désormais sous les auspices d'une négociation peu transparente et corporatiste, que le scandale des logiciels de moteurs, destinés à cacher leur pollution réelle, a mis en évidence. La démission de M. Hartz, puis de son 
PDG à une date plus récente, ne s'accompagne au demeurant d'aucune réforme structurelle interne.

Le nom de Macron est désormais associé à une gestion néocapitaliste de la crise économique globale. Le discours ministériel est idéologiquement significatif, il troque le prolétariat contemporain contre la classe créative, il préfère les entrepreneurs aux ouvrières illettrées, il avantage les grandes surfaces plutôt que les caissières, les licenciements plutôt que leur contrôle, appelle à la concurrence libre plutôt qu'au socialisme, et la concurrence libre à la place de l'internationalisme. Cette idéologie est médiatiquement surévaluée en ce qu'elle assemble des stéréotypes, des injonctions morales et des argumentaires conservateurs. La même idéologie est dans le même temps lourdement sous-estimée par des gauches intellectuelles qui se contentent de la dénoncer, au lieu de comprendre la vérité déformée qu'elle exprime, tout comme le gaullisme, la social-démocratie, le communisme ou le fascisme exprimèrent des réalités vécues, de manière détournée. La nouvelle loi de la mondialisation capitaliste, ses normes et son principe directeur ne vont pas se dissoudre à la faveur de discours patriotiques et républicains, charitables, identitaires ou modernisateurs.

L'interprétation que je viens de défendre est que le principe Macron résume une stratégie de codification juridique du capitalisme concurrentiel à l'échelle mondiale, qui s'applique à l'Europe en tant qu'ensemble continental avec le concours actifs de tous les Etats membres. L'OMC, le FMI, les traités libre-échangistes internationaux trouvent leur application européenne dans la flexicurité, de même que la législation Hartz allemande qui est érigée en modèle à suivre par les apologues du principe Macron. Le terme composé, la flexi-curité, peut être décrypté sur la base de notre interprétation. La flexibilité qui est l'adaptation au jeu de la concurrence nécessite une nouvelle base juridique qui en garantisse le cadre, une forme de pouvoir durable et une psychologie de masse susceptible de le supporter. Il ne s'agit pas de contourner l'Etat et les organisations de masse au profit d'un libéralisme sans loi, mais bien au contraire de transformer toute l'organisation sociale et sa représentation, grâce au concours de l'Etat et des organisations qui y participent. Ne pas tourner le regard analytique vers ce problème revient à le détourner, sinon de mener des charges contre des moulins à vent.

\section{Chantiers et champs de lutte}

24 Afin de montrer l'étendue, la cohérence et l'imbrication des chantiers de la bureaucratie liquide, à partir du principe précis de la flexicurité, je vais caractériser la loi travail (El Khomri) avant de souligner sa correspondance dans les grands traits avec la législation Hartz allemande, ce qui fait apparaître des champs de lutte généraux en Europe. Je rappelle que la loi travail tout comme la législation Hartz sont des transpositions de recommandations écrites de la Commission européenne à ses Etats membres, et que ces lois s'inscrivent dans les directives-cadres européennes relatives à la concurrence libre.

Les principaux aspects et objectifs de la flexicurité que je vais commenter sont "l'entreprise qui respire" et qui licencie (accords d'entreprise); le temps de travail sans fin ni répit; le marché du travail comme domaine de la précarité à vie; le contrôle administratif des allocataires et demandeurs d'emploi. Enfin, nous verrons les lignes de clivage et de conflit qui esquissent les champs de lutte contemporains, loin de la 
rhétorique fasciste, souverainiste ou social-libérale qui occupe l'espace public bourgeois en France.

\section{La loi travail française}

L'opposition à la loi travail française - qui est entrée en vigueur sous une forme amoindrie comparée à son ambition initiale en janvier 2017 - s'est focalisée sur les modalités de licenciement, la précarisation des contrats de travail, la baisse de la rémunération des heures supplémentaires, la défense des conventions collectives, statuts et prérogatives syndicales, et dans une mesure moins centrale, le temps de travail et les accords d'entreprise dans les secteurs où les conventions jouent un rôle faible ou insignifiant. C'est sur ce dernier point que le mouvement a subi une défaite politique, à côté de victoires incontestables qui se manifestent dans le retrait de plusieurs dispositions du projet de loi au printemps qui concernent les modalités et motifs de licenciement en particulier. D'autres succès du mouvement concernent le maintien de conventions collectives qui étaient promises à la désintégration dans les transports, routiers et SNCF en tête, ainsi que le régime des Intermittents du spectacle. Aussi, la convocation d'un comité de refonte du droit du travail qui devait généraliser et amplifier la loi travail, fut abandonnée en mars 2017 au vu de l'atmosphère hostile dans les rangs de la gauche syndicale, parlementaire et extra-parlementaire. De manière globale, le mouvement a favorisé la recrudescence locale et sectorielle de grèves et luttes depuis l'été 2016, il a réalisé un retournement idéologique majeur dans l'espace militant, syndical, partidaire, universitaire, et dans l'électorat potentiel de la gauche, retournement qui se mesure dans l'éviction politique de l'exécutif HollandeValls, sans même nommer l'intégralité des sondages d'opinion qui ont massivement désavoué le principe même de la flexicurité que porte la loi travail.

Avant de revisiter des principes-clé de la loi, voyons ce que la loi ne dit pas, ses non-dits pour parler comme Sigmund Freud. Deux chapitres de la loi, son introduction philosophique et le renvoi direct au rapport Badinter, furent abandonnés dès le début des hostilités pour tenter de sauver l'essentiel, alors qu'elles rendent explicites le projet politique global. Le chapitre initial de la loi travail, dit "exposé des motifs", témoigne lumineusement de la volonté de s'inscrire dans la nouvelle loi de la mondialisation capitaliste et son idéologie, son monde. L'exposé dit, au nom de M. Le Premier Ministre E. Valls : "Le monde du travail entre dans une phase de profonds changements. Les dernières décennies n'en ont pas été exemptes : elles ont été marquées par la mondialisation, la part croissante des services dans notre économie et l'élévation des qualifications. (...) Le projet de loi visant à instituer de nouvelles libertés et de nouvelles protections pour les entreprises et les actifs doit permettre une refondation de notre modèle social. La démarche de refondation est triple : - elle concerne d'abord le code du travail. Les principes essentiels du droit du travail, dégagés par le comité présidé par Robert Badinter, serviront de base à une réécriture du code selon une nouvelle architecture en trois parties (...), les règles d'ordre public auxquelles il n'est pas possible de déroger ; le champ renvoyé à la négociation collective ; les règles supplétives applicables en l'absence d'accord. Le présent projet de loi met dès à présent en place cette nouvelle architecture pour la partie du code, relative au temps de travail et aux congés. Il crée une commission de refondation chargée de mener ce travail à son terme, dans un délai de deux ans. Elle devra renforcer la place de la négociation collective, notamment au niveau de l'entreprise." ${ }^{139}$ 

à la mondialisation capitaliste, et qui permet aux patrons de décider au niveau de leur propre entreprise de réduire les droits et rémunérations des salariés concernés sans leur accord. L'article $1 \mathrm{du}$ rapport Badinter, ancien garde des Sceaux dont le nom qui semble convoquer l'esprit des droits de l'homme, précise que "Les libertés de la personne sont garantis", sauf si elles sont limités par "les nécessités du bon fonctionnement de l'entreprise". En somme, le droit du travail en tant que droit collectif n'existe pas, et les droits de l'homme peuvent être réduits en fonction des besoins du capital dans l'entreprise. "Liberté, Egalité, Bentham" écrit Marx dans Le Capital pour se moquer du théoricien des libre-échangistes de son époque. Désormais vaut : "Liberté, Badinter, Bentham". De manière aussi pamphlétaire et pertinente à la fois, Emmanuel Todd, supporteur du candidat Hollande, a saisi le sens de son action une fois parvenu à son poste présidentiel : Hollande serait en train de "détruire le prolétariat". ${ }^{40}$

\section{d'actualité, enterrées avec le comité de refondation du droit du travail qui devait suivre l'application de la loi.}

Dans un registre plus factuel, précisons que la loi prévoyait jusqu'alors que la norme légale est le CDI et que la norme du temps de travail qui s'y applique est de 35 heures par semaine, sans détour (art. 13). Il faut ainsi constater que le principe de la loi travail impose l'accord d'entreprise au détriment du code du travail, de la norme légale et du droit collectif. Auparavant, le code primait sur la convention, qui devait être autorisée par le gouvernement élu via son Ministère compétent, et tous deux primaient sur tout accord d'entreprise qui ne pouvait en aucun cas limiter ou détériorer les droits des salariés. Désormais, un accord d'entreprise peut augmenter le temps de travail jusqu'au maximum de 60 heures hebdomadaires prévues par la directive cadre européenne de 2003 (ou 48 heures en moyenne dans la durée), abolir les jours non travaillés, fériés, weekends, récupération compris, diminuer le paiement des heures théoriquement supplémentaires à partir de la 35ème heure, et diminuer les congés. Par cet acte, qui constitue une intervention inédite dans le droit du travail collectif des salariés, inégalée depuis la Libération du nazisme, la loi travail impose la priorité à la règle entrepreneuriale. Le gouvernement français appelle ce renversement violent "l'inversion de la hiérarchie des normes". L'inversion s'apparente à une révolution néoconservatrice. Même le gouvernement allemand qui a établi la législation Hartz dans les années 2000 n'a jamais osé renverser le droit du travail à ce point, car il se serait heurté à une opposition frontale du syndicalisme de masse unifié qui compte encore 8 Millions d'adhérents. En effet, dans le droit allemand, aucun accord d'entreprise ne peut sous-passer les normes légales ni les conventions collectives de branche. De surcroit, aucun accord d'entreprise ne peut être conclu séparément au sein d'un même groupe ou d'une même firme, car cet accord nécessite alors la mise en place d'une convention collective à part entière qui doit elle-même être conforme à une loi fédérale qui régit ce cas de figure particulier, tout en respectant l'intégralité de la législation générale du travail. ${ }^{41}$ Dire que la loi travail s'adosse à l'expérience allemande est particulièrement mensonger sur ce point. L'influence syndicale sur les conventions collectives, qui coiffent les accords d'entreprise en Allemagne, entrave et ralentit encore considérablement l'imposition de normes patronales spécifiques dans chaque entreprise qui se fait au gré des situations concurrentielles, au point que de 
nombreuses entreprises ont décidé des contourner l'obstacle en se quittant les conventions pour appliquer directement la loi qui peut se montrer plus rigide. Environ la moitié des salariés allemands est couverte par les conventions collectives.

De la même manière, en France, le maintien de conventions collectives sectorielles qui assurent une certaine cohérence des situations et des différents métiers concernés, parfois issues d'anciennes entreprises nationalisées (Orange, EDF, etc.), bloquent la généralisation de l'accord d'entreprise voire des accords de site localisés, ce qui fait que la règle concurrentielle ne s'impose pas pleinement en tant que nouvelle norme. D'où les tentatives et attaques répétées des gouvernements, Valls en tête, qui consistent à dissoudre ces conventions collectives spécifiques et les statuts comparables des Intermittents, Universitaires et autres fonctionnaires du secteur territorial, hospitalier, etc. Même dans les domaines qui concernent au premier chef les accords d'entreprise de dumping social, à savoir l'industrie et le commerce, des résistances et inerties fortes bloquent la règle concurrentielle. De tels accords dérogatoires sont possible et prévus depuis la seconde loi Aubry au sujet des 35 heures (l'un des exemples fut l'accord Bosch de Vénissieux), puis ils furent encouragés par un accord national interprofessionnel transposé dans une loi en 2015, mais en pratique, peu d'entreprises sont parvenues à imposer des accords défavorables aux salariés. Le regain actuel de combativité des salariés sur un plan local ou sectoriel, et une certaine radicalisation syndicale que cela favorise, visible au sein de la CGT, doublé de recours en justice, neutralise encore la capacité de nuisance du principe concurrentiel-légal.

L'accord d'entreprise concurrentiel est au cœur du renversement légal, en ce qu'il incarne la bureaucratie liquide d'une organisation managériale qui impose sa loi sans jamais rendre des comptes ni endosser une responsabilité institutionnelle. A côté de ce noyau dur de la loi, défini dans sa seconde section, d'autres sections concernent la formation professionnelle (subordonnée aux besoins immédiats des entreprises tout comme l'est le droit du travail, au lieu d'être qualifiante de manière globale), la facilitation du licenciement, la médecine du travail allégée, et enfin la question européenne irrésolue des travailleurs détachés.

La loi travail est flanquée d'autres lois et dispositifs qui visent une recomposition d'ensemble : la redéfinition légale du temps de travail au niveau national et européen, l'invention légale de nouveaux types de contrats précaires (à un Euro, à zéro heure, à l'essai pour des durées prolongées, le Minijob, le complément au RSA, etc.); la réorganisation centrale et autoritaire de la gestion des demandeurs d'emploi et de leurs allocations, du point de vue du travail des conseillers et du point de vue de l'imposition de nouvelles conditions d'attribution d'emplois aux chômeurs, au rabais.

Pour saisir la cohérence et l'obstination des politiques menées sur le plan européen par le blairisme, en particulier en France et en Allemagne, cette comparaison structurelle des chantiers et champs de lutte est utile. Pour cela, il s'agit de ne pas s'arrêter sur les intitulés enjoliveurs, scintillants ou trompeurs des projets et lois, qui peuvent d'ailleurs changer de titre au cours de leur réalisation. La loi Macron fut initialement intitulée "loi Noé", puis une autre loi Macron fut attribuée à la Ministre El Khomri et son intitulé officiel a changé, passant de "loi pour les nouvelles libertés et nouvelle protections des entreprises et actifs" à "nouvelles protections des salariés", afin de gommer l'idéologie explicite de l'activation qui fait partie de la flexicurité. Aussi, l'ANI (accord national interprofessionnel) est devenu la loi sur la sécurisation de l'emploi. 
Le tableau suivant donne une vue d'ensemble à la cohérence programmatique de mesures et lois qui sont appliquées dans une dispersion apparente, en France et en Allemagne, et qui vont toutes dans le même sens unilatéral de la flexicurité au détriment du droit collectif des salariés et demandeurs d'emploi.

\begin{tabular}{|c|c|c|}
\hline $\begin{array}{l}\text { Chantier / champ de } \\
\text { lutte }\end{array}$ & France & Allemagne \\
\hline $\begin{array}{l}\text { Précarisation des } \\
\text { contrats de travail }\end{array}$ & $\begin{array}{l}\text { Loi El Khomri; } \\
\text { CDD renouvelable } 3 \text { fois } \\
\text { Extension des périodes d'essai }\end{array}$ & $\begin{array}{l}\text { Hartz } 2 \\
\text { CDD renouvelable } 3 \text { fois } \\
\text { Jobs à } 400 \text { Euros } \\
\text { Extension de la période à l'essai } \\
\text { en CDI }\end{array}$ \\
\hline $\begin{array}{l}\text { Contrôle et activation } \\
\text { des chômeurs }\end{array}$ & $\begin{array}{l}\text { PARE (1999); RSA; accord Unedic 2015, } \\
\text { cassé par le conseil d'Etat, mais repris } \\
\text { dans la loi El Khomri }\end{array}$ & $\begin{array}{l}\text { Hartz } 4 \\
\text { Jobs à } 1 \text { Euro }\end{array}$ \\
\hline $\begin{array}{l}\text { Désorganisation de la } \\
\text { norme collective du } \\
\text { temps de travail et des } \\
35 \text { heures }\end{array}$ & $\begin{array}{l}\text { Loi Aubry } 2 \text { - } \\
\text { métallurgie 1999; décrets Fillon de } \\
\text { non-taxation } 4 \text { des heures } \\
\text { supplémentaires; ANI / loi séc. emploi; } \\
\text { loi El Khomri; accords dans } \\
\text { l'automobile d'allongement du temps } \\
\text { de travail sans augmentation de } \\
\text { salaire }\end{array}$ & $\begin{array}{l}\text { Pacte pour l'emploi et la } \\
\text { compétitivité (1995); convention } \\
\text { collective nationale VW 1995; } \\
\text { Agenda } 2010 \text { Schröder; } \\
\text { application par des accords du } \\
\text { maximum prévu par la directive } \\
\text { européenne de 2003:60h }\end{array}$ \\
\hline $\begin{array}{l}\text { Imposition de l'accord } \\
\text { d'entreprise contre la } \\
\text { convention collective } \\
\text { et la loi }\end{array}$ & $\begin{array}{l}\text { ANI; loi de sécurisation de l'emploi; loi } \\
\text { El Khomri }\end{array}$ & $\begin{array}{l}\text { Conventions collectives de la } \\
\text { métallurgie depuis 1996, principe } \\
\text { dérogatoire repris par l'ensemble } \\
\text { des branches }\end{array}$ \\
\hline $\begin{array}{l}\text { Affaiblissement des } \\
\text { droits syndicaux et des } \\
\text { institutions issues du } \\
\text { mouvement ouvrier }\end{array}$ & $\begin{array}{l}\text { Loi Macron; seuils pour constituer des } \\
\text { comités d'entreprise ou CHSCT, } \\
\text { affaiblissement de l'inspection du } \\
\text { travail }\end{array}$ & $\begin{array}{l}\text { Augmentation du nombre de } \\
\text { salariés pour la constitution des } \\
\text { différents types de représentation } \\
\text { des salariés / CE }\end{array}$ \\
\hline $\begin{array}{lll}\text { Mise en question } & \mathrm{du} \\
\text { service public } & \mathrm{de} \\
\text { l'emploi } & & \end{array}$ & $\begin{array}{l}\text { Fusion ANPE-Assedic vers le Pôle } \\
\text { emploi (2008) } \\
\text { Contrôle direct des demandeurs } \\
\text { (mesure Rebsamen) } \\
\text { Radiations facilitées } \\
\begin{array}{l}\text { Projets de dégressivité et } \\
\text { conditionnalité des allocations } \\
\text { chômage }\end{array}\end{array}$ & $\begin{array}{l}\text { Désorganisation du service public } \\
\text { général (Hartz 1) } \\
\text { Délocalisation et privatisation } \\
\text { partielle des agences pour } \\
\text { l'emploi Job centers } \\
\text { Conditionnalité de ressources, } \\
\text { sanctions financières, radiations } \\
\text { faciles }\end{array}$ \\
\hline
\end{tabular}




\section{La législation Hartz}

37 La réforme du marché de l'emploi qu'il a impulsé se décline en quatre lois votées en 2003-04 (Hartz I à IV) qui couvrent les champs essentiels de la flexicurité : création d'agences privées de recherche d'emploi (Hartz I), changement des règles contractuelles et introduction de nouveaux emplois à faible revenu dont le Minijobs, etc. (Hartz II), changement du statut du service public de l'emploi (Hartz III) ; fusion des régimes d'assurance chômage et de l'aide sociale comportant un dispositif renforcé de sanctions (Hartz IV).

La loi Hartz 1 désorganise l'ancien service public de l'emploi, au profit d'un système de management semi-privé. Le mécontentement des usagers, rebaptisés clients, et les nombreuses plaintes des agents ont provoqué une révision de ces procédures, qui fut encouragée par le mouvement social à l'encontre du principe Hartz en 2004. Le caractère bureaucratique de la gestion des clients a été décrit minutieusement par des chercheurs, tandis que les expériences sensibles et expressions subjectives des chômeurs furent recueillies par Brigitte Valenthin. ${ }^{42}$

De manière complémentaire, la loi Hartz IV organise la fusion des allocations chômage et l'attribution des autres aides sociales, dans un même système d'activation et de sanction des chômeurs ${ }^{43}$. L'indemnisation du chômage est limitée à un an (ALG I), après lequel joue le régime minimal ALG II, dont l'intitulé populaire est justement « Hartz 4 » en référence à la loi qui l'encadre. Dans sa première version, la loi ne prend pas en compte la durée de la période de cotisation à l'assurance chômage, ce qui annule par exemple l'ancienneté des chômeurs âgés. Surtout, la loi impose la liquidation de toute autre ressource privée (biens immobiliers, épargne, etc.) de la chômeuse ou du chômeur et de son conjoint, avant le premier versement de l'allocation ALG II. Depuis, les gouvernements Merkel ont rétabli un certain principe d'ancienneté et ont défini le droit à des biens à hauteur de 50.000 Euros. C'est probablement la dureté de ces mesures qui a provoqué l'un des plus importants mouvements sociaux en Allemagne depuis l'après guerre, d'autant plus remarquable qu'il ne fut pas soutenu par les directions syndicales du DGB.

La transformation de la frustration en réussite, ou alors de la motivation en échec, que réalise le principe Hartz est en particulier apparue dans notre évaluation du programme Innopunkt dans le Brandebourg. ${ }^{44}$ Le programme, financé grâce au fonds de développement régional de l'UE, est contrôlé selon un principe managérial (log-frame) très similaire à celui en vigueur sur le plan intergouvernemental. Il s'agit d'un management par objectifs chiffrés, en l'occurrence du nombre de chercheurs d'emploi qui ont participé avec succès à des projets de formation et de réinsertion. Parmi les chômeurs participants, certains ont retrouvé un emploi salarié qui ne leur permettait pas d'achever leur formation au sein des projets, ce qui fut apprécié comme une réussite personnelle par les intéressés. Du point de vue de l'évaluation quantitative des projets, ce succès devait pourtant être acté comme un abandon de projet, donc un échec. Systématiquement, le général prime sur le particulier, pour paraphraser Adorno, et la politique publique ne peut être évaluée à partir de l'expérience vécue des sujets, mais doit être interprétée en fonction des classifications pré-établies (le log-frame). De la même manière s'organise la définition des demandeurs d'emploi en catégories basées sur une supposée « employabilité » au sein des agences pour l'emploi sous le régime 
Hartz, sachant que les demandeurs qui ont trouvé des emplois qualifiés étaient souvent issues de catégories à faible employabilité.

41 Afin de compléter la perception des salariés du processus dont il est question, il est saisissant de lire ou d'écouter les expressions des fonctionnaires chargés de les accueillir qui décrivent leur malaise face à des personnes officiellement définies en tant que "clients". Lors de notre évaluation du projet brandebourgeois de réinsertion des chômeurs de longue durée très qualifiés, nous avons fait parler des femmes et hommes de plus de cinquante ans qui se sentaient infantilisés par un système de classement des agences pour l'emploi qui définit avant tout leur "proximité en rapport au marché de l'emploi" plutôt que de leurs qualifications et expériences professionnelles. Dès lors que des formateurs les interrogeaient sur leurs capacités personnelles, cela fut souvent perçu comme une "éclosion" ou une "renaissance", termes récurrents dans nos entretiens ouverts avec les chômeurs, et proches d'un imaginaire lié à l'expérience sensible.

Au cours de nos nombreux entretiens avec des agents de pôle emploi dans cinq villes tunisiennes, en $2009{ }^{45}$ nous avons rencontré des phénomènes comparables. Il va de soi que les règles des agences pour l'emploi furent établies selon le modèle français, jusque dans l'organisation spatiale des bureaux, et que la flexicurité européenne en constitue le cadre programmatique via les accords de coopération internationaux (Banque mondiale/Banque européenne de développement/Euroméditerranée). Lors d'un entretien semi-directif, une conseillère d'emploi qui a fondu en larmes alors qu'elle cherchait à nommer son impuissance, face à la condition économique et institutionnelle qui semble sans issue, à la veille de la révolution: "On fait tout, courrier, administrateur, submergé par d'autres choses qui le décalent et qui l'empêchent de faire l'initiative et de trouver des issues. Le rôle est important mais il faut faire plus et il faut changer! C'est une mentalité et je dis qu'il faut changer (...) il faut une autre mentalité".

Il ne manque à cet agenda de flexibilisation de l'emploi que le domaine du temps de travail, qui n'est pas réglementé de manière stricte par la loi en Allemagne, mais dont l'application flexible dépend des entreprises. M. Hartz a inauguré un nouveau type d'accord d'entreprise dérogatoire en tant que DRH de Volkswagen dans les années 1990, qui a largement relativisé les standards collectifs, contenus dans les conventions collectives de branche.

En Allemagne, le bilan empirique concret et illustré de la législation Hartz a mis en évidence toutes les limites du principe Hartz. En effet, le marché du travail allemand atteint un pic d'emplois atypiques mis en place par les lois Hartz, alors que les salaires sont globalement bas, tandis que la flexibilité des horaires de travail progresse et que l'encadrement par les conventions collectives s'affaiblit. La progression de la flexicurité n'a manifestement pas d'effet causal sur les indicateurs de croissance. Les chiffres statistiques présentés par des auteurs favorables à l'Agenda 2010 montrent une baisse réelle des salaires sur la période de son application via les lois Hartz, ${ }^{46}$ fait qui a procuré un avantage aux entreprises exportatrices allemandes au sein de la concurrence intraeuropéenne. Les seuls chiffres statistiques qui peuvent être directement reliés aux lois Hartz proviennent du recensement des nouveaux types de contrats de travail précaires qui ont été autorisés par cette législation, par exemple les jobs à 400 Euros par mois (exonérés d'impôt) ou à 1 Euro par heure (en complément des allocations chômage). Ces formes de contrat précaire ont explosé, faussant par la même occasion la 
perception du chômage de masse puisque ces mini-jobs sortent du taux de chômage. L'emploi précaire a atteint $40 \%$ de la population active en 2014 , dix ans après la législation Hartz.$^{47}$ En ce sens, ces lois ont accompagné à la marge la baisse salariale voulue par le gouvernement Schröder, puisqu'elles ont entraîné une baisse des rémunérations dans le segment le plus précaire du marché du travail. Dans la conception de Peter Hartz, cela correspond à l'objectif d'établir un "secteur de bas salaires" pour des services nécessitant des qualifications faibles. Dans les faits, cet objectif fut atteint, avec des salaires horaires entre 4 et 7 Euros dans certains domaines. Cela a provoqué l'apparition d'une couche de travailleurs pauvres et l'extension de la pauvreté à environ 6 millions de personnes si on englobe les familles et enfants, cela concerne aujourd'hui un quart de la population active.

Plusieurs articles et quelques rapports de recherche ont tenté de saisir la dynamique des mouvements de contestation qui se sont formés à l'encontre du principe Hartz et la flexicurité. ${ }^{48}$ Nous retenons la révolte contre la législation Hartz en 2004 qui a mobilisé au moins 250.000 citoyens. Cet auteur a découvert que la majorité de citoyens mobilisés à travers des réunions publiques et des manifestations régulières du lundi appartenaient aux classes moyennes, alors que les chômeurs et précaires directement concernés sont présents dans une moindre mesure, soutenus localement par des cortèges ouvriers organisés. L'association publique de groupes sociaux divers indique l'émergence d'un espace public oppositionnel. Un exemple comparable fut le mouvement hexagonal contre le Contrat première embauche en 2006. Parti de nombreuses occupations d'universités, la mobilisation a touché des secteurs publics et finalement les salariés du privé. Les interprétations normatives varient fortement, alors qu'Honneth qualifia le mouvement contre le CPE de « lutte des travailleurs pour la reconnaissance ", Alain Bertho a voulu y voir la "dernière révolte fordiste", mais celle-ci fut suivie par une nouvelle révolte encore plus large au printemps 2016, dix ans plus tard. ${ }^{49}$ Le nombre de manifestants a dépassé un million à partir du 31 mars 2016, à plusieurs reprises, les grèves ont touché sinon paralysé de nombreux secteurs dont les plus classiques (transports, ports et docks, raffineries), mais aussi la jeunesse scolarisée et étudiante. Le mouvement s'est exprimé à travers un espace public oppositionnel aux multiples expressions médiatiques et politiques : la pétition en ligne et en masse (attribuée à C. de Haas), les vidéos youtube (p.ex. On vaut mieux que ça) et mobilisations informationnelles via les réseaux sociaux; les Assemblées générales de grève locales ou interprofessionnelles, les manifestations unitaires, les blocus de collèges, lycées et universités, les blocages physiques, les occupations de sites et de dépôts, l'occupation de la place de la République et d'autres lieux transformés en forum démocratique en dehors des institutions étatiques (Nuit debout). Le nombre de grèves locales et sectorielles a sensiblement augmenté après et malgré la fin du mouvement à l'encontre de la loi travail, depuis l'automne 2016 jusqu'en 2017, comme le signale Jacques Chastaing, qui mène un travail remarquable de recensement des conflits de travail en l'absence de toute statistique officielle, travail que les services du ministère de l'emploi ne fournissent plus. L'auteur analyse les informations disponibles dans la presse régionale, afin de lister et documenter le nombre de grèves sur un blog. Les seuls mois de janvier et février 2017 font apparaitre plus d'un million de jours de grève cumulés, et ne prennent pas en considération la grève générale guyanaise d'avril 2017. ${ }^{50}$ Le fait d'ignorer les bilans statistiques désastreux de la flexicurité, ou le fait de faire disparaitre les formes de sa contestation actuelle ne sauraient amoindrir la force de la critique. 


\section{Bilan et perspectives}

Pourquoi François Hollande, son ancien Ministre Macron qui fut son secrétaire général au palais de l'Elysée, et leurs alliés ont-ils voulu s'entêter à suivre une voie et un principe qui a fait la preuve générale de son échec, sinon de sa faillite sur le plan européen, local et moral? La thèse de l'obstination idéologique, de la psychorigidité, émerge alors presque spontanément. A ce propos, Paul Krugmann, prix Nobel d'économie a proclamé dès janvier 2014 "l'effondrement intellectuel" de François Hollande et de ses soutiens, ${ }^{51}$ mais l'économiste devrait savoir que son échec prend racine dans celui de la famille Clinton qu'il a soutenu en 2016 en la personne d'Hillary Clinton. Il ne s'agit pas du problème d'un homme ou d'une femme, mais d'un programme, d'une stratégie internationale et d'une loi générale de la mondialisation capitaliste.

Le programme, la stratégie et la loi de la mondialisation capitaliste, furent portés de la manière la plus cohérente par un bloc démocrate international qui vient de se disloquer sous nos yeux. Ce bloc fut formé par le gros de la social-démocratie européenne, lancé par la famille Clinton, les amis d'A. Blair et les blairistes français dont F. Hollande et affiliés -, il fut accompagné par les directeurs M. Strauss Kahn au FMI, P. Lamy à l'OMC, M. Macron au secrétariat général de l'Elysée, les premiers ministres Ayrault et Valls, soutenu par les rapporteurs J. Attali, E. Besson et R. Badinter, les économistes libéraux du type A. Tyrole, des sociologues rocardiens du style P. Rosanvallon, le philosophe J. Habermas qui présida le club européen de D. Strauss Kahn et qui fut le seul à être cité dans le livre de campagne de M. Hollande, avant de supporter E. Macron. Ce même bloc fut encore composé par la Cfdt depuis Nicole Notat, par le Medef et un grand nombre de petits ou grands élus allant du socialisme verbal au centre-droit démocrate et chrétien, qui forment le socle de la bureaucratie liquide, et qui ne proposent aucun bilan écrit ni prise de responsabilité pour la situation actuelle. Une situation marquée par une montée des inégalités sans précédent en Europe et en Amérique, notamment entre hommes et femmes, entre riches et pauvres, entre détenteurs de capitaux et salariés ou demandeurs d'emploi, vieux et jeunes, entre régions industrialisées et marginalisées, entre bourgeois et prolétaires. Une situation qui stimule d'énormes mouvements oppositionnels dans les pays fondateurs de l'UE, sinon des insurrections civiques ou des révolutions politiques aux marges de l'Europe, comme en Islande, Bosnie ou Tunisie. Une situation qui favorise en même temps une radicalisation à droite selon le schéma classique de la psychologie de masse décrit par Freud, Reich, Fromm et Adorno. Une situation enfin qui dissipe la rhétorique conservatrice de la fin de l'histoire, de l'absence de lutte des classes et de l'arrêt du clivage historique droite/gauche, désiré par les théoriciens nazis à commencer par Carl Schmitt, contesté par les évènements. Après avoir été neutralisé par un discours centriste qui stipula que "la lutte des classes n'existe pas" (J. Cahuzac), les courants de gauche européens qui se réfèrent explicitement aux mouvements oppositionnels contre la loi capitaliste et bureaucratique qui prévaut actuellement sont en passe de remplacer la vielle gauche blairiste. Ce mouvement fut amorcé en Grèce avant de se répandre à travers toute l'Europe du Sud, depuis le Portugal et l'Espagne. Aujourd'hui, il est en train de gagner la gauche en France, en Europe de l'Est, sinon en Allemagne où la 
social-démocratie imagine pour la première fois de se démarquer publiquement du programme Hartz qui a causé sa perte durable dès 2004.

L'Agenda européen, allemand et français qui est parti du sommet de Lisbonne fut un échec complet selon les propres critères que la Commission européenne s'était assignée. Au lieu du plein emploi, le chômage de masse a explosé pour atteindre des sommets historiques encore jamais atteints, les reprises partielles se sont accompagnées d'une vaste extension de la précarité qui avoisine la moitié de la population active en Allemagne. L'inégalité entre femmes et hommes s'est accrue, de même qu'entre les générations, les niveaux de qualification. Les inégalités, entre régions européennes industrialisées et désindustrialisées, riches et pauvres, se sont approfondies. L'Agenda 2010 des socialistes, démocrates et centristes qui se retrouvent aujourd'hui dans le principe Macron a ainsi radicalisé les inégalités de classe et de genre, déployant en pratique son message idéologique, bureaucratique, anti-marxiste, anti-intellectuel et anti-féministe. L'exécutif Valls-Hollande, soutenu et relayé par E. Macron lors des présidentielles de 2017, a illustré ce message à merveille, entre invectives à l'encontre du syndicalisme ouvrier, provocations explicites à l'encontre de la CGT et de la gauche extra parlementaire, un mépris de classe affirmé à l'égard des "sans dents", des femmes prolétaires de Gad qui seraient des illettrées, la suspicion collective jetée sur les sans emploi qui ne voudraient pas travailler, et une ode à la gloire de l'idiotie à l'encontre des chercheuses et chercheurs en sciences humaines qui excuseraient le terrorisme islamique par leur simple pensée analytique et critique.

Le bilan factuel de cet agenda européen, dont les statistiques prouvent le caractère néfaste, concorde in fine avec le discours idéologique de ses défenseurs, et qui est teinté d'un néoconservatisme compatible avec les droites européennes. Ce discours, son programme et ses élites représentatives sont désavoués par l'électorat de gauche, l'espace syndical européen, les jeunes générations et une intelligentsia critique ascendante. Ces peuvent se souvenir les fondements d'une approche cosmopolitique et internationaliste, issue des révolutions européennes et du renversement des traditions impériales, coloniales et capitalistes.

Les possibilités de la critique, cet élan qui promet de défaire la bureaucratie liquide qui pèse encore sur les imaginaires et les pratiques tel un cauchemar, ne résident pas dans l'imploration impuissante des institutions qui ont fait la fortune des dominants actuels, mais dans une approche qui sorte du cadre établi. Max Weber avait brillamment montré que le principe d'organisation des nouveaux et vieux partis ouvriers avait pour point d'aboutissement ultime la bureaucratisation, la bureaucratie sans démocratie. Le PS en apporte la confirmation historique. Aujourd'hui, il s'agit de reprendre la lutte antibureaucratique que mena le premier cercle des amis et discutants de Weber, composé d'anarchistes et de marxistes inclassables (Michels, Trotski, Lukacs, etc.), il s'agit de sortir de la tutelle et de la servitude volontaire pour avancer à nouveau sur le chemin droit de l'émancipation, comme l'a signalé le philosophe allemand de la révolution française, Emmanuel Kant..$^{52}$ Avancer, loin de la malveillante et fausse traduction de Weber à qui l'on voudrait attribuer une fausse neutralité en lieu et place de la liberté (Wertfreiheit). Le chemin de la rectitude se situe encore et depuis toujours à gauche. Dit avec les mots de Walter Benjamin dans ses Thèses sur l'histoire, cette conscience qui se rend compte qu'il s'agit toujours de faire exploser le continuum de l'histoire appartient aux classes révolutionnaires (thèse XV). Au moment où plus aucun 
ordre institutionnel traditionnel ne tient, face au principe de la bureaucratie liquide, qui est intenable, cet adage s'applique pleinement.

\section{NOTES}

1. Le présent texte s'inspire d'une conférence d'A. Neumann qui fut prononcé dans l'Amphi X de l'Université Paris 8 en avril 2016, intitulé "Le pouvoir de l'esprit contre l'esprit du pouvoir", à l'invitation du comité de grève de l'Université, occupée pour obtenir le retrait intégral de la loi travail. Le texte est appelé à se poursuivre sous la forme d'un essai plus ample dans la collection $\mathrm{K}$ des éditions Terra.

2. Y. Varoufakis, Le minotaure planétaire, Le Cercle, 2014; Y. Varoufakis, Notre printemps d'Athènes, Les liens qui libèrent, 2015.

3. U. Huws, «La crise comme aubaine sans précédent pour le capitalisme: une nouvelle accumulation par la marchandisation des services publics », Variations n.18, mai 2013.

4. Les documents confidentiels qui notent l'avancée de négociations ont été publiés sous la forme d'une fuite par la section neerlandaise de Greenpeace en 2016 : https://ttip-leaks.org/ttip/ national-treatment-and-market-access-for-goods/

5. Bilan documenté de manière détaillée dans Th. Frank, Listen, Liberal, MetropolitainBooks, 2016; K. Dixon, Les évangélistes du marché, Raisons d'agir, 2008; B. Sanders, Our Revolution, Dunne Books, 2016.

6. Le président Hollande a ainsi fait ratifier le Traité européen d'union budgétaire dès l'année de son investiture en 2012, et qui fut co-rédigé par des chefs d'Etat de droite (Sarkozy, Merkel) et sociaux-démocrates (Faymann, Monti, etc.).

7. En témoigne l'opposition tardive mais explicite de la confédération syndicale allemande DGB à l'Agenda 2010, la mise en question rhétorique de cette politique par le candidat social-démocrate à la Chancellerie, M. Schulz, ou encore l'effondrement du parti social-démocrate néerlandais PvdA qui a perdu les deux tiers de son électorat aux législatives de 2017 après avoir co-gouverné avec la droite.

8. A. Touraine, La voix et le regard, Seuil, 1978, p.26 en accès libre: http://psychaanalyse.com/pdf/ la_voix_et_le_regard_alain\%20_touraine_1978_317pages.pdf

La césure historique du mouvement ouvrier occidental de 1978 fut diagnostiqué à la suite des défaites des grandes grèves dans l'industrie automobile à l'instar de la Fiat italienne, dans la sidérurgie allemande, puis des mineurs anglais, et qui trouvent une correspondance dans des victoires électorales néoconservatrices (Thatcher, Barre, Reagan, Kohl). Touraine et le rocardisme ont théorisé ces échecs pour écarter toute approche qui examine les capacités d'action du prolétariat, voir : A. Neumann, "Travail, activité, activation" in Sociologie et sociétés, n. $48,2016$.

9. Les syndicats explicitement opposés à la loi travail (CGT, FO-CGT, CGC, Solidaires, FSU) représentent la grande majorité des salariés du pays aux élections professionnelles de 2017, et la CGT arrive en tête sur la plan national avec 1,9 millions de voix (source : Ministère du travail).

10. En témoigne le succès en librairie de titres publiés par des éditeurs indépendants, la relance des libraires alternatifs, le succès numérique de revues critiques démontré par les statistiques en ligne (à titre d'exemple, Variations est passé de 2000 lecteurs lors de l'élection de Sarkozy en 2007 à 40.000 par an en 2016), le succès de la presse critique, entre Mediapart et le Monde 
diplomatique, ou des nombreux blogs et chaines youtube qui se réfèrent au mouvement dont il est question.

11. Cet article fut rédigé et acté avant les présidentielles de 2017 et fait l'économie d'une analyse électorale approfondie.

12. W. Streeck est passé de l'apologie du corporatisme concurrentiel dans la mondialisation capitaliste à sa critique souverainiste : Streeck, Der Korporatismus in Deutschland, Campus, 1999; Streeck, Du temps acheté, Gallimard, 2014. U. Beck a glissé de la société du risque vers la dénonciation du social-darwinisme : Beck, La société du risque, Champs Flammarion, 2008; Beck, Entretien à la Frankfurter Rundschau, 5/02/2010.

13. Appel publié dans Le Monde du 2 décembre 1995 http://www.liberation.fr/cahier-special/ 1998/01/12/contre-l-archaisme-pour-une-reforme-de-fond-de-la-securite-sociale_544820

14. J. Habermas, La constitution de l'Europe, Gallimard, 2013; voir A. Neumann, Après Habermas, Delga, 2015. Pour la position de Rosanvallon, voir le débat avec $\mathrm{S}$. Wahnich publié par Mediapart en 2015 : https://www.youtube.com/watch?v=dusR-b99J5U

15. K.Marx, Das Kapital, 1, Dietz Verlag, 1979, pp.761-802.

16. A titre d'exemple, voir A. Honneth, Die Idee des Sozialismus, Surhkamp, 2015. Il s'agit d'un essai qui reprend l'intégralité des positions politiques d'E. Durkheim à l'encontre de Marx et le mouvement socialiste de son époque, à comparer avec l'essai posthume de Durkheim, Le socialisme, 1928 : http://classiques.uqac.ca/classiques/Durkheim_emile/le_socialisme/ le_socialisme.pdf

17. Z. Baumann, Liquid modernity, Polity Press Cambridge, 2000.

18. Th. Adorno, La dialectique négative, Payot, 2003; W. Benjamin, Thèses sur l'histoire, Folio, 2004; Negt, L'espace public oppositionnel, Payot, 2007; Negt/Kluge, Geschichte und Eigensinn, Steidl, 2016; N. Fraser, Le féminisme en mouvements, La Découverte, 2009; Becker-Schmidt/Knapp, Feministische Theorien, Campus, 2004.

19. M. Maffesoli, défenseur du postmodernisme français, avait assorti cette thèse d'un pari, dans ses Sarkologies sociologiques, au moment de l'élection présidentielle de 2012 : puisque la société française serait devenue post-moderne, le candidat qui incarne cette posture devrait être élu, Nicolas Sarkozy. Pari perdu.

20. M.Weber, Ökonomie und Gesellschaft, Zweitausendeins, 2000.

21. H. Ford, The international Jew (1920), Fq Classics, 2007.

22. I. Bruno, Déchiffrer l'Europe compétitive: Etude du benchmarking comme technique de coordination intergouvernementale dans le cadre de Lisbonne, 2006.

23. www.oecd.org/LongAbstract/0,3425,en_2649_34141_34227699_119684_1_1_1,00.html

24. A. Neumann, Review of the Working Time Directive 2003 - Case Study Germany, Economisti associati, 2011.

25. Dardot/Laval, La nouvelle raison du monde, La Découverte, 2009.

26. J.M. Vincent, Le mouvement ouvrier en Allemagne de l'Ouest, Thèse de doctorat, Fondation de sciences politiques, Paris, 1963; P. Von Oertzen, Betriebsräte in der Novemberrevolution. Eine politikwissenschaftliche Untersuchung über Ideengehalt und Struktur der betrieblichen und wirtschaftlichen Arbeiterräte in der deutschen Revolution 1918/19. Droste, Düsseldorf, 1963; Von Oertzen, Hartz - Demontage des Sozialstaats , VSA, Hambourg, 2004; P. Bourdieu et alli, Les perspectives de la protestation, Syllepse, 1998.

27. Voir l'article d'A. Le Marchand au sein de ce dossier thématique de la revue Variations (n.20).

28. A. Neumann, Après Habermas, Delga, 2015; Neumann, Kritische Arbeitssoziologie, Schmetterling, (2ème édition) 2016; Conscience de casse, ed. Variations / La Quatrième Génération, 2010.

29. C. Castoriadis, L'expérience du mouvement ouvrier, 10/18, 1979.

30. D. Graber, Bureaucratie, Les liens qui libèrent, 2015.

31. http://www.reseau-terra.eu/article1330.html

32. https://www.tagesschau.de/inland/hartzvierurteil102.html 
33. Commission européenne, Lisbon Strategy Evaluation document, Bruxelles, 2010, p.6.

34. Y. Moulier-Boutang, Le capitalisme cognitif, Amsterdam, 2009.

35. Isabelle Bourgeois, "Le réformes Hartz, remise en cause de l'Etat social?", Regards sur l'économie allemande, 2013, référence électronique Isabelle Bourgeois, « Les réformes Hartz, remise en cause de l'Etat social ? ", Regards sur l'économie allemande $n .108,2013$.

36. Boltnski/Chiappello, Le nouvel esprit du capitalisme, Gallimard, 1999.

37. Rucht/Yang: "Wer demonstrierte gegen Hartz IV?» in Forschungsjournal Neue Soziale Bewegungen, Heft 4, Berlin, 2004, p. 21-27.

38. http://www.spiegel.de/jahreschronik/a-521375.html

39. Projet de loi visant à instaurer de nouvelles libertés pour les entreprises et les actifs, Assemblé nationale, mars 2016.

40. E. Todd, Goodbye Hollande!, Marianne, 4/05/2013

41. Voir le texte de loi original : Bundestarifgesetz de 1959, https://www.bgbl.de/xaver/bgbl/ start.xav?

startbk=Bundesanzeiger_BGBl\&jumpTo=bgbl159s0313.pdf\#__bgbl__\%2F\%2F*\%5B\%40attr_id\%3D\%27bgbl159s0313.pdf\%27\%5D__1489942

42. Brigitte Valenthin, Ich bin dann mal Hartz 4,VSA, Hambourg, 2010.

43. Peter von Oertzen, Hartz - Demontage des Sozialstaats, VSA, Hambourg, 2004.

44. Alexander Neumann, Begutachtung der Innopunkt Initiative Ältere - Erfahrung trifft auf Herausforderung, Entwurf zum Endbbericht für das Arbeitsministerium Brandenburg, 2010, 60 p.

45. Alexander Neumann, Organisation reform of Aneti Tunisia, Banque mondiale, 2009 (l'annexe comporte 50 pages de retranscription anonymisée d'entretiens, rapport confidentiel).

46. M. Burda, "The German Miracle", SFB, 2017 https://sfb649.wiwi.hu-berlin.de/papers/pdf/ SFB649DP2016-005.pdf

47. Voir la fondation scientifique du syndicalisme allemand, HBS, https://www.boeckler.de/ wsi_5859.htm

48. D. Rucht, op.cit.

49. Axel Honneth, Le Monde, 2.4.2006.; Alain Bertho, «Du grondement de la bataille à l'anthropologie du contemporain » in Variations n.8, Vs/Parangon, Lyon, 2006.

50. J. Chastaing, https://blogs.mediapart.fr/jean-marc-b/blog/270317/un-tournant-dans-lasituation-sociale-par-jacques-chastaing

51. P. Krugmann, "The intellectual collapse of François Hollande", The New York Times, 1/2014.

52. J.M. Vincent, Max Weber ou la démocratie inachevée, Felin, 1998; M. Löwy, Rédemption et utopie, éditions du Sandre, 2009; I. Kant, Qu'est-ce que les Lumières?, 1784; W. Benjamin, Le concept d'histoire, Folio, 2004.

\section{INDEX}

Mots-clés : Bureaucratie liquide, loi travail, flexicurité, Emmanuel Macron, mondialisation capitaliste, Théorie critique 
AUTEUR

\section{ALEXANDER NEUMANN}

Professeur en sciences de la communication à l'Université Paris 8 - Vincennes, Cemti, directeur de publication de Variations 